\title{
Representações Sociais da Hipertensão Arterial
}

\author{
Mariana Luíza Becker da Silva ${ }^{1}$ \\ Andréa Barbará da Silva Bousfield \\ Departamento de Psicologia da Universidade Federal de Santa Catarina, \\ Florianópolis, SC, Brasil
}

\begin{abstract}
Resumo
A hipertensão arterial sistêmica (HAS) é responsável por significativo número de óbitos. O controle pode ser medicamentoso ou/e por hábitos saudáveis, os quais representam uma das principais dificuldades, tendo em vista o envolvimento com a ideia de saúde. Com isso, vislumbra-se a relevância da Teoria das Representações Sociais (TRS), pois é possível conhecer as facilidades e dificuldades no tratamento, para planejar intervenções na formulação de políticas públicas. A presente pesquisa visou identificar as representações sociais da HAS e de seu tratamento para pessoas com esse diagnóstico. Participaram 20 moradores de Florianópolis - 10 homens - com média de idade 54 anos e 9 meses $(D P=7$ anos e 10 meses). Foram realizadas entrevistas em profundidade e o material foi submetido a três análises hierárquicas descendentes (CHD), pelo software IRAMUTEQ. Uma relacionada à HAS, outra ao tratamento e outra unindo as duas temáticas. Na primeira análise apareceram as causas, preocupações, sintomas, rotina e processo da descoberta. Na segunda, surgiram relatos sobre à aquisição do remédio, importância, vantagens e desvantagens do tratamento, alimentação, preocupações e rotina. Na última, percebe-se os conteúdos já mencionados e o entrelaçamento da HAS como doença e de seu tratamento. Pode-se afirmar, que tratamento e doença se inter-relacionam.
\end{abstract}

Palavras-chave: Representações sociais, hipertensão arterial, tratamento.

\section{Social Representations of Arterial Hypertension}

\begin{abstract}
Arterial hypertersion is responsible by significant number of death. The control can be medicinal or/and for healthy habits, which represent one of the principal difficults, for as much us the evolvement with health's idea. With this, catch a glimpse of the importance of Social Representation Theory, so is possible to know the facilities and difficulties in the treatment, plan interventions in the formulation of public politics. The aim was identify the social representation of arterial hypertension and your treatment for people with this diagnosis. Participated 20 residents in Florianópolis, 10 men, with on an average 54 and 9 months years old ( $S D=7$ years and 10 months). Realized interviews in depth and the material was submited the three hierarchic analysis descendants (CHD), by software IRAMUTEQ. One relacionated the hypertasion, treatment and other joing the two tematics. In the first analysis appeareded the causes, preocupations, symptons, routine and process of the discovery. In the second, appeareded reports about the purchase of the medicine, importance, advantages and disadventages of the treatment, alimentation,
\end{abstract}

Endereço para correspondência: Delminda Silveira, 568, Apto 208, Agronômica, Florianópolis, SC, Brasil 88025-500. Fone: (048) 9964-6850. E-mail: marianaluiza_b@hotmail.com e andreabs@gmail.com

Financiamento: Programa Institucional de Bolsas de Iniciação Científica - Conselho Nacional de Desenvolvimento Científico e Tecnológico (CNPq). 
preocupation and routine. In last perceive the contents mentionated before and the interlacing of the hypertersion, how disease and your treatment. We can affirmed that treatment and disease interrelate.

Keywords: Social representations, arterial hypertension, treatment.

\section{Representaciones Sociales de la Hipertensión Arterial}

\section{Resumen}

Hipertensión arterial sistémica (HAS) es responsable por un número significativo de muertes. Control puede ser médico o hábitos saludables, que es una dificultad importante, en vista de la implicación con idea de salud. Vislumbra la relevancia de Teoría de la Representación Social (TRS), es posible saber las comodidades y dificultades en tratamiento, para planificar intervenciones en políticas públicas. El objetivo fue identificar representaciones sociales de la hipertensión y su tratamiento para las personas con este diagnostico. El estudio incluyó 20 sujetos que viven en Florianópolis - 10 - hombres con edad media de 54 años y 9 meses ( $S D=7$ años y 10 meses). Realizaron entrevistas en profundidad y el material fue sometido a tres análisis de clasificación jerárquica descendientes (CHD) por medio de software IRAMUTEQ. Una relacionada con hipertensión arterial, tratamiento y otra que unen los dos. El primer análisis apareció causas, preocupaciones, síntomas, rutina y proceso de descubrimiento. La segunda, surgieron informes sobre adquisición del remedio, importancia, ventajas y desventajas del tratamiento, alimentos, preocupaciones y rutina. En el final, apareció el contenido ya mencionado y el entrelazamiento de hipertensión como una enfermedad y su tratamiento. Puede decir al respecto, que tratamiento y enfermedad están relacionados entre sí.

Palabras clave: Representaciones sociales, hipertensión arterial, tratamiento.

As doenças crônicas não transmissíveis são caracterizadas por múltiplos fatores de risco, períodos de latência extensos, curso prolongado, e origem não infecciosa (Ministério da Saúde, 2011). Dentre elas está a hipertensão arterial sistêmica (HAS), a qual apresenta um aumento significativo todo ano e seu aparecimento é cada vez mais precoce. A HAS é uma condição clínica multifatorial caracterizada por uma pressão arterial sistólica maior ou igual a $140 \mathrm{mmHg}$ e uma pressão arterial diastólica maior ou igual a $90 \mathrm{mmHg}$, em pessoas que não fazem uso de medicação anti-hipertensiva. A sua presença propicia outras enfermidades, associa-se frequentemente a alterações funcionais e/ou estruturais dos órgãos-alvo e a alterações metabólicas, com consequente aumento do risco de eventos cardiovasculares fatais e não-fatais (Sociedade Brasileira de Cardiologia, Sociedade Brasileira de Hipertensão, \& Sociedade Brasileira de Nefrologia 2010).

A hipertensão arterial é responsável por um grande número de óbitos em todo o mundo. No
Brasil, conforme as IV Diretrizes Brasileiras de Hipertensão (Sociedade Brasileira de Cardiologia et al., 2010), a HAS afeta mais de 30 milhões de brasileiros e é o maior fator de risco para o desenvolvimento de outras doenças. Segundo Schmidt e col. (2011) 72\% das mortes em 2007 foram motivadas por doenças crônicas. Dados do Ministério da Saúde (2012), mostram que até outubro de 2012, haviam no estado de Santa Catarina 316.703 casos de hipertensão arterial, desconsiderando os casos com diabetes associada. Estima-se que 25,5\% dos homens e 20,7\% das mulheres com idade igual ou superior a 18 anos tenham diagnóstico prévio de hipertensão arterial. Já para as pessoas com 50 anos ou mais, essa estimativa aumenta para aproximadamente $50 \%$ dos homens e mais de $50 \%$ das mulheres.

A HAS é caracterizada tanto como uma doença, quanto como um fator de risco e agravo para outras enfermidades. Além disso, ela não possui cura, e demanda um tratamento que se prolonga por toda a vida. A partir disso, o conhecimento existente a respeito da HAS e as medidas 
de controle constituem-se em elementos capazes de reduzir drasticamente óbitos decorrentes desta doença, desde que aplicados corretamente em sua totalidade (Santos, Frota, \& Cruz, 2005).

A prática de controle dessa doença, silenciosa e progressiva, pode ser medicamentosa, por meio do uso de drogas específicas receitadas pelo médico; e (ou) não medicamentosa, quando envolve a modificação do estilo de vida da pessoa e adoção de comportamentos mais saudáveis. Quando não tratada adequadamente, a hipertensão arterial pode acarretar graves consequências a alguns órgãos vitais. Com isso, se constitui em um dos mais graves problemas de saúde pública. Estima-se que $40 \%$ dos acidentes vasculares encefálicos e que $25 \%$ dos infartos ocorridos em pacientes hipertensos poderiam ser prevenidos. No entanto, por ser na maior parte do seu curso assintomática, uma parcela importante da população adulta não sabe que é hipertensa, e muitos dos que sabem não estão sendo adequadamente tratados (Ministério da Saúde, \& Organização Pan Americana de Saúde, 2004). A falta de aderência ao tratamento é uma grande preocupação para o sistema de saúde visto que menos de $1 / 3$ dos hipertensos adultos tem sua pressão arterial adequadamente controlada (Leão e Silva et al., 2013; Péres, Magna, \& Viana, 2003).

As modificações de estilo de vida são de fundamental importância no processo terapêutico e na prevenção da hipertensão, sem o qual os medicamentos não resultarão em níveis adequados de pressão arterial. Apesar de ser uma condição crônica sem cura, ela pode ser estabilizada com a adoção de práticas de controle adequadas e modificação dos comportamentos de risco (Ministério da Saúde, 2011; Schmidt et al., 2011; Sociedade Brasileira de Cardiologia et al., 2010). Alimentação adequada, sobretudo quanto ao consumo de sal, dieta com frutas, hortaliças, fibras e minerais, controle do peso, prática de atividade física, controle do estresse psicossocial, controle do tabagismo e uso excessivo de álcool são fatores que devem ser adequadamente abordados e controlados (Ministério da Saúde, 2006; Pérez Riera, 2000; Sociedade Brasileira de Cardiologia et al., 2010). Além disso, a idade, o sexo, a etnia, fatores socio-econômicos, gené- ticos e de contexto influenciam na prevalência da HAS (Ministério da Saúde, 2006; Sociedade Brasileira de Cardiologia et al., 2010).

As mudanças de comportamento aparecem como as principais dificuldades na efetivação do tratamento, tendo em vista que a adesão à terapêutica é deficitária, principalmente quando estabelecidos durante um longo tempo de vida (Figueiredo \& Asakura, 2010; Péres et al., 2003). Para Jardim, Souza e Monego (1996), modificar o estilo de vida envolve mudanças na forma de viver e na própria ideia de saúde. A concepção de saúde é formada por meio da vivência e pela experiência pessoal, tendo estreita relação com suas crenças e valores. Acredita-se também que as crenças conforme as pessoas tendem a viver afetam diretamente os hipertensos na forma como enfrentam a doença e o tratamento da mesma. Percebe-se com isso, a necessidade da compreensão acerca da dimensão representacional da hipertensão arterial, aprofundando seu significado construído e partilhado socialmente pelas pessoas.

A educação em saúde é apontada por Péres et al. (2003) como uma das formas para estimular a adesão ao tratamento. Conforme Spink (1992) é necessário para isso conhecer os costumes sobre práticas de saúde, os valores e as percepções do paciente que são muitas vezes diferentes daqueles pensados pelos profissionais da saúde. Torna-se necessário conhecer e considerar as práticas populares de saúde para uma maior adesão ao tratamento. A partir disso, Leão e Silva et al. (2013) apresentam a relevância da Teoria das Representações Sociais (TRS) no estudo da HAS. Tal teoria fornece instrumentos de identificação de conceitos possibilitando trabalhar com o pensamento social em sua dinâmica e em sua diversidade.

A TRS compreende-se em um conjunto de conceitos, ideias e explicações sobre a realidade, sendo originadas na vida cotidiana, a qual é refletida nas formas particulares de uma sociedade adquirir conhecimento sobre o mundo (Moscovici, 1982). Além disso, estabelece-se como uma forma de conhecimento, que é produzido e partilhado pelas pessoas com o objetivo de construir uma realidade comum aos membros 
de um grupo (Jodelet, 2001). Moscovici (2010) expõe que as representações sociais tem como função permitir às pessoas orientarem-se em seu meio social, e, além disso, construir uma rede de significados que favoreçam a comunicação sem ambiguidade. Em conformidade Jodelet (2001) explica que as representações sociais orientam e organizam as condutas e as comunicações.

As Representações Sociais segundo Moscovici (1978) podem ser descritas como verdadeiras teorias do senso comum, "ciências coletivas", pelas quais se constroem à interpretação e as realidades sociais. Estudar as representações sociais é identificar as visões de mundo que os indivíduos ou grupos possuem e este se reflete nas representações sociais do objeto, as quais operam como agentes de influência nas práticas sociais dos sujeitos em um contexto de produção de sentidos (Chaves \& Silva, 2011; Morin, 2004).

Nesse sentido, Oliveira e Zanetti (2011) afirmam que a TRS contribui no conhecimento sobre o que favorece e o que dificulta a mudança de estilo de vida. Apreende-se com isso o processo pelo qual os sujeitos constroem o pensamento que circula em torno da doença, e que age como fator de influência na percepção de necessidades específicas à saúde, e consequentemente na representação do próprio tratamento. Dessa forma, segundo Abric (1998) as representações sociais se tornam prescritivas, servindo como um direcionamento para as ações sociais.

Entendendo a relevância dos estudos referentes às representações sociais, Morin, Apostolidis, Dany, Preau, e Spire (2007) argumentam a utilização da TRS no campo da saúde, em que deve-se considerar os diferentes pontos de vista acerca do objeto saúde e doença. Em conformidade, Badziak e Moura (2010) entendem que a compreensão sobre o que é saúde varia em diferentes grupos sociais e também varia dentro do mesmo, assim é necessário conhecer o real significado de saúde para esta população antes de desenvolver uma política de saúde. Dessa forma valoriza-se o conhecimento do senso comum como campo de significação da realidade, produção de saberes e encontro entre os atores sociais que compõem essa relação (Morin et al., 2007). Mantovani et al., (2008) em uma pesquisa realizada com hipertensos demonstrou que os usuários de uma forma geral, possuíam informações objetivas sobre a doença, porém a condição crônica é um fator que não é claro para esse grupo, sendo constatado que alguns usuários afirmam que ela possui cura. A atribuição dessa cura era direcionada a aspectos metafísicos, como divindades, magia ou ainda a um programa de tratamento bem realizado.

Um aspecto a ser considerado é o marco de mudança na vida diária, refletida principalmente nas restrições à dita vida "normal", provocando sentimentos negativos frente ao tratamento (Costa e Silva et al., 2008; Costa e Silva \& Moura, 2011; Mantovani et al., 2008). Com isso, é necessário a reflexão sobre como é viver com uma doença crônica, tendo em vista suas características restritivas. Outro fator de destaque é o aspecto emocional. Esse elemento figura como um forte fator de influência sobre a adoção de práticas de controle da doença (Costa e Silva et al., 2008; Costa e Silva \& Moura, 2011; Péres et al., 2003). Em conformidade, Boell, Meirelles, Silva e Lessmann (2012) verificaram que quando o estresse foi reconhecido como um fator de influência, os pacientes sentiram-se mais compreendidos e mais favoráveis ao diálogo. Nesse sentido, a intervenção profissional pode ser beneficiada com a compreensão das representações, pois a valorização dos saberes do senso comum é decisiva para pensar a tomada de decisão dos programas de saúde (Galand \& Salès-Wuillemin, 2009).

O processo comunicacional desempenha um papel determinante na circulação das informações, uma vez que estas irão compor o conhecimento da pessoa que vive com hipertensão sobre sua própria condição. Costa e Silva e Moura (2011) em sua pesquisa descrevem que apesar dos profissionais de saúde desenvolverem ações informativas relacionadas às práticas de controle da hipertensão, os usuários acabavam agindo por seus conhecimentos informais, demonstrando uma não absorção dos conhecimentos científicos divulgados pelos profissionais. 
Compreender esse contexto possibilita à ciência, disponibilizar conhecimentos mais aprimorados sobre uma determinada ocorrência, gerando condições mais favoráveis para aplicação de estratégias de intervenção junto à população. Com isso, o presente estudo visa realizar um levantamento das representações sociais que se relacionam com a hipertensão arterial e com o seu tratamento. Conhecer e compreender os aspectos acima mencionados pode fornecer subsídios para a elaboração e/ ou aperfeiçoamento de políticas públicas eficientes e mais assertivas no combate à doença.

\section{Método}

Trata-se de uma pesquisa de caráter descritiva e exploratória, do qual participaram 20 sujeitos que vivem com a hipertensão arterial - 10 homens e 10 mulheres (média de idade 54 anos e 9 meses, $D P=7$ anos e 10 meses) . Para a seleção dos participantes utilizou-se a técnica de amostragem por bola de neve (D’Ancona, 1996), a qual consiste em um participante indicar a outro, que indica outro e assim sucessivamente. Como critério de inclusão, os participantes deveriam ter mais de 30 anos, considerando que há maior prevalência de hipertensão arterial a partir dessa idade (Sociedade Brasileira de Cardiologia et al., 2010) e viver com HAS por no mínimo um ano. Como critérios de exclusão, participantes com diabetes associada ou algum tipo de transtorno grave que impossibilitasse a interação com o pesquisador.

A coleta de dados foi feita por meio de entrevistas em profundidade, a qual utilizou-se a técnica da entrevista não direta (Ghiglione \& Matalon, 1993), sobre a temática da hipertensão e o seu tratamento. Além disso, havia questões sociodemográficas e de caracterização para contextualizar o sujeito em seu contexto social. O roteiro de entrevista foi baseado em dados da literatura e na realização de entrevistas no pré-teste. As entrevistas foram gravadas e posteriormente transcritas (média de tempo de 40 minutos).

Posteriormente organizou-se o material textual decorrente da entrevista em três corpus separadamente, um apenas em relação às representações sociais da hipertensão arterial, outro envolvendo as representações do tratamento da hipertensão arterial e por último um corpus envolvendo os dois temas (representação na doença e representações do tratamento) em duas subtemáticas distintas. Essa divisão foi realizada a fim de compreender melhor a relação entre a representação da doença e a representação do tratamento.

O conteúdo textual contido nos três corpus foram submetidos cada um à uma análise hierárquica descendente, por meio do software IRAMUTEQ (Camargo \& Justo, 2013; Ratinaud \& Marchand, 2012). Além disso, as questões sociodemográficas foram submetidas à análise descritiva no software SPSS (Statistical Package for the Social Science - Pacote Estatístico para Ciências Sociais), versão 20.0.

A pesquisa teve a aprovação do Comitê de Ética para Pesquisa com Seres Humanos (Parecer 242.982 e CAAE: 11917113.3 .0000 .0121$)$ e foi realizada com base nos determinantes instituídos pela resolução 196/1996 e 466/2012 do Conselho Nacional de Saúde - CNS.

\section{Resultados}

Participaram das entrevistas 20 pessoas, sendo 10 homens, os quais possuem entre 36 e 68 anos, a média de idade foi de 54 anos e 9 meses ( $D P=7$ anos e 10 meses). Verificou-se que 13 participantes possuem uma alta escolaridade (ensino superior completo ou incompleto), 5 participantes possuem um nível médio de escolaridade (ensino médio completo) e 2 dos participantes possuem um baixo nível de escolaridade (ensino fundamental completo ou incompleto). Em relação a renda familiar, 8 participantes possuem renda entre $\mathrm{R} \$ 3501,00$ a $\mathrm{R} \$ 6000,00,6$ dos participantes possuem renda maior que $\mathrm{R} \$ 6.001,00,4$ possuem renda inferior a $\mathrm{R} \$ 3500,00$ e 2 não responderam.

A quantidade de tempo que foram diagnosticados com hipertensão arterial variou entre $1 \mathrm{e}$ 25 anos, sendo que 8 participantes descobriram a doença entre 1 e 4 anos, 5 participantes entre 
5 e 9 anos e 7 descobriram a mais de 10 anos. Observou-se também que 11 participantes são casados ou estão em uma união estável, 8 são divorciados e 1 viúvo. A quantidade de filhos variou entre nenhum e 5 , sendo a média de 2 filhos.

O primeiro corpus a ser analisada pelo software IRAMUTEQ foi sobre as representações sociais da doença (hipertensão arterial). A partir da Classificação Hierárquica Descendente (CHD) foram analisados 357 segmentos de texto (ST), retendo $89,70 \%$ do total, gerando 5 cinco classes. O software dividiu o corpus Hipertensão em dois subcorpora por meio da CHD conforme a Figura 1.
O primeiro sub-corpus, composto pela classe 1 e 2, apresenta uma relação com o passado e com o futuro, as causas que provocaram a hipertensão e as consequências que podem surgir sem o devido cuidado. O segundo sub-corpus formado pelas classes 3 , 4 e 5, se relacionam pela narrativa das próprias histórias de vida dos sujeitos, como descobriram a doença, os sintomas que estavam presentes e os sentimentos relacionados à descoberta. O software ainda dividiu esse $s u b$ -corpus novamente opondo a classe 5 às classes 3 e 4 . Na classe 5 trata-se mais sobre o histórico dos sintomas sentidos diferenciando-se dos temas abordados nas classes 3 e 4 referentes mais sobre o processo da descoberta.

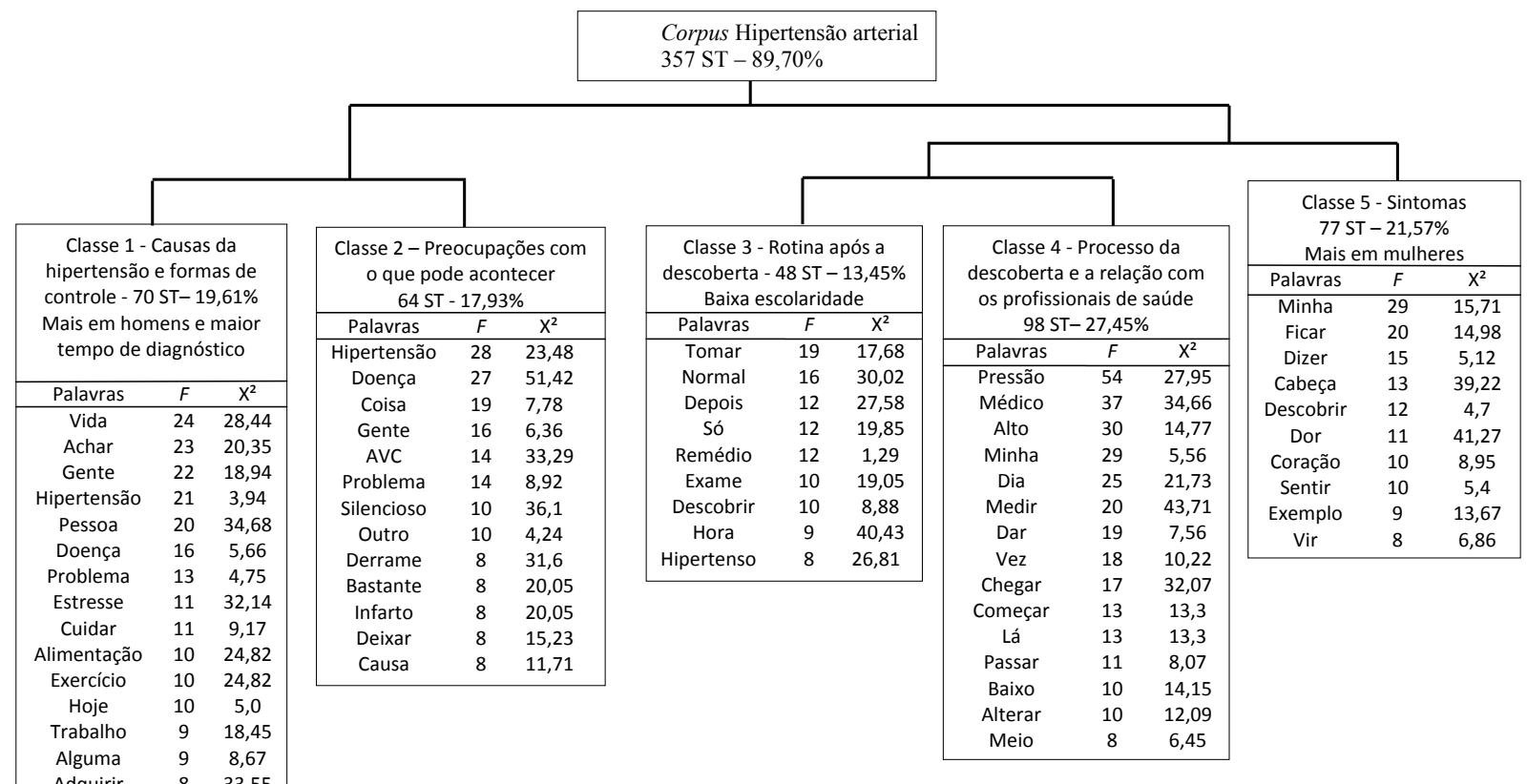

Figura 1. Dendograma da Classificação Hierárquica Descendente das representações sociais da hipertensão arterial.

A classe 1 chamada de "Causas da hipertensão e formas de controle" foi responsável por $19,71 \%$ dos segmentos de texto. Apareceu predominantemente nas falas de homens com um maior tempo de diagnóstico. Os principais elementos que se associaram a essa classe foram: vida, pessoa, estresse, alimentação, exercício, trabalho, cuidar, entre outras. O conteúdo desta classe trata principalmente das concepções das causas da hipertensão e de modos e formas que podem ser controlada a mesma. $\mathrm{O}$ trecho a seguir ilustra esse contexto:

...É dessa forma que eu estou combatendo e estou me livrando dessa doença eu acredito eu quero muito que eu pare com a medicação já que eu adquiri a hipertensão por causa de um estresse muito grande que eu tive na minha vida pessoal e profissional. (Participante 2, sexo masculino, tempo de diagnóstico 2 anos)

A classe 2 chamada de "Preocupações com o que pode acontecer" foi responsável por 17,93\% 
dos segmentos de texto. Os principais elementos que se associaram a essa classe foram: doença, hipertensão, AVC, silencioso, problema, derrame, entre outras. O conteúdo desta classe trata principalmente das preocupações relacionadas à doença, o que pode acontecer caso a pessoa com hipertensão não se cuide, as consequências da hipertensão. $\mathrm{O}$ trecho a seguir ilustra esse contexto: "Você pode ter um AVC, você pode ter um infarto e não pode brincar porque é uma doença e nem todo mundo está ciente disso, eu era uma pessoa que não levava a sério" (Participante 9, sexo feminino, tempo de diagnóstico 2 anos).

Em seguida aos desdobramentos, a classe 5 chamada de "Sintomas" foi responsável por $21,57 \%$ dos segmentos de texto. Apareceu predominantemente nas falas das mulheres. Os principais elementos que se associaram a essa classe foram: minha, cabeça, dor, ficar, coração, entre outras. O conteúdo desta classe trata principalmente dos sintomas sentidos no processo da descoberta da doença. O extrato a seguir ilustra esse contexto: "Tem muita gente que tem dor na nuca tem muita gente que tem alteração aqui nessas veias do pescoço e tem gente que não sente nada" (Participante 5, sexo feminino, tempo de diagnóstico 10 anos).

A classe 3 chamada de "Rotina após a descoberta" foi responsável por $13,45 \%$ dos segmentos de texto. Apareceu predominantemente nas falas de pessoas com baixa escolaridade. Os principais elementos que se associaram a essa classe foram: tomar, normal, remédio, exame, hora, entre outras. O conteúdo desta classe trata principalmente do convívio com a doença após a descoberta dela. $\mathrm{O}$ trecho a seguir ilustra esse contexto: "Só em uma conversa ele já percebeu que eu estava com pressão alta então ele já fez os exames e verificou, depois dessa data até agora o remédio continuou diário e não tem mais volta" (Participante 3, sexo masculino, tempo de diagnóstico 6 anos).

A classe 4 chamada de "Processo da descoberta e a relação com os profissionais de saúde" foi responsável por $27,45 \%$ dos segmentos de texto. Os principais elementos que se associaram a essa classe foram: pressão, médico, dia, medir, entre outras. O conteúdo desta classe trata de como foi a descoberta da doença, a relação direta com médicos, exames de rotina, medição da pressão e no acompanhamento que se tem a partir da descoberta, como pode ser exemplicado: "Descobri nas consultas de rotina mesmo eu ia fazer e começou a dar alterado então a médica pediu para eu fazer um controle, medir todo dia no mês para ver como ela ia se portar" (Participante 15, sexo feminino, tempo de diagnóstico 2 anos).

Analisando o dendograma e o conteúdo obtido pela análise desse primeiro corpus, verifica-se uma noção de hipertensão arterial associada as causas que provocaram a doença e as consequências que podem surgir sem o devido cuidado, perpassadas pelas narrativas das próprias histórias de vida dos sujeitos, como descobriram a doença, os sintomas que estavam presentes, os sentimentos vivenciados e as mudança na rotina envolvendo o controle medicamentoso para evitar complicações.

O segundo corpus a ser analisado pelo software IRAMUTEQ foi sobre as representações sociais do tratamento da hipertensão arterial. A partir da CHD foram analisados $506 \mathrm{ST}$, retendo $75,07 \%$ do total, gerando 5 cinco classes. O software dividiu o corpus Tratamento em 2 subcorpora, conforme a Figura 2.

O primeiro sub-corpus formado pela classe 5 , traz questões mais externas ao indivíduo, culpabiliza-se o governo por não dar a devida atenção à saúde de pessoas que não podem comprar a medicação que é cara. Já o Segundo sub-corpus, composto pela classe 1, 4, 2 e 3 trazem questões associadas à pessoa, particularidades relacionadas ao seu modo de vida, as vantagens e desvantagens que acreditam possuir o tratamento, os sintomas e as preocupações advindas, o histórico da descoberta e as consequentes mudanças na rotina, incluindo alimentação, exercício físico, a medicação, entre outros. O software dividiu esse sub-corpus novamente opondo a classe 1 às classes 2, 3 e 4 . Na classe 1 trata-se mais sobre aspectos da opinião dos participantes a acerca da importância e as vantagens e desvantagens de seguir o tratamento, diferenciando-se dos temas 
abordados nas classes 2, 3 e 4 referentes mais sobre a rotina e os sentimentos de preocupação com as consequências caso não adaptem a rotina ao tratamento adequado. E ainda subdividiu a classe 4 às classes 2 e 3 . Na classe 4 trata-se mais sobre aspectos da história da descoberta da doença, assim como situações de mudança na vida a partir do tratamento, separando-se das classes 2 e 3 referentes à preocupação com os sintomas e com a alimentação.

\begin{tabular}{|c|c|c|c|c|c|c|c|c|c|c|c|c|c|c|}
\hline & \multirow{2}{*}{\multicolumn{2}{|c|}{4.}} & \multirow{6}{*}{\multicolumn{3}{|c|}{$\begin{array}{c} \\
\text { Classe } 4 \text { - Alimentação } \\
72 \text { ST - 14,23\% } \\
\text { Pouco tempo de diagnóstico, } \\
\text { pouca escolaridade, + em } \\
\text { mulheres }\end{array}$}} & \multicolumn{3}{|c|}{$\begin{array}{l}\text { Corpus Tratamento } \\
506 \text { ST- } 75,07 \%\end{array}$} & & & & & & \\
\hline & & & & & & & & & & & & \multirow{2}{*}{\multicolumn{3}{|c|}{$\begin{array}{c}\text { Classe } 5 \text { - Aquisição do } \\
\text { remédio - } 120 \text { ST - } 23,72 \% \\
\text { + tempo de diagnóstico + } \\
\text { mulheres }\end{array}$}} \\
\hline \multirow{3}{*}{\multicolumn{3}{|c|}{$\begin{array}{c}\text { Classe } 1 \text { - Importância, } \\
\text { vantagens e desvantagens } \\
127 \text { ST - } 25,1 \% \\
\text { Pouco tempo de diagnóstico } \\
+ \text { em homens }\end{array}$}} & & & & \multirow{4}{*}{\multicolumn{3}{|c|}{$\begin{array}{c}\text { Classe } 2 \text { - Sintomas e } \\
\text { preocupaç̃̃es } \\
92 \text { ST - 18,18\% } \\
\text { Baixa escolaridade + homens }\end{array}$}} & \multirow{3}{*}{\multicolumn{3}{|c|}{$\begin{array}{c}\text { Classe } 3 \text { - Depois da } \\
\text { descoberta como ficou a rotina } \\
95 \mathrm{ST}-18,77 \%\end{array}$}} & & & \\
\hline & & & & & & & & & & & & Palavras & $F$ & $X^{2}$ \\
\hline & & & & & & & & & & & & Remédio & 40 & 10,08 \\
\hline Palavras & $F$ & $\mathrm{X}^{2}$ & & & & & & & Palavras & $F$ & $\mathrm{X}^{2}$ & Pessoa & 30 & 11,67 \\
\hline Tratamento & 51 & 83,94 & Palavras & $F$ & $\mathrm{X}^{2}$ & Palavras & $F$ & $\mathrm{X}^{2}$ & Achar & 31 & 12,69 & Comprar & 25 & 52,85 \\
\hline Seguir & 34 & 91,89 & Remédio & 24 & 5,38 & Pressão & 50 & 115,72 & Gente & 26 & 4,53 & Mês & 19 & 31,92 \\
\hline Só & 24 & 3,99 & Ficar & 20 & 10,7 & Tomar & 45 & 30,38 & Sempre & 21 & 34,08 & Morrer & 17 & 30,92 \\
\hline Cuidar & 23 & 42.34 & Coisa & 20 & 6,3 & Assim & 31 & 28,82 & Minha & 21 & 8,0 & Acabar & 16 & 23,59 \\
\hline Nenhum & 23 & 29,17 & Sal & 19 & 104,36 & Pensar & 19 & 27,96 & Dizer & 19 & 27,78 & Conseguir & 16 & 14,21 \\
\hline Doença & 22 & 22,04 & Já & 17 & 7,09 & Medicamento & 18 & 19,84 & Ano & 18 & 31,46 & Saúde & 16 & 8,41 \\
\hline Dificuldade & 22 & 22,04 & Comer & 14 & 72,68 & Alto & 17 & 33,82 & Dia & 18 & 10,15 & Posto & 15 & 49,72 \\
\hline Vida & 20 & 6,55 & Deixar & 14 & 35,25 & Dia & 17 & 8,75 & Vida & 17 & 8,43 & Caro & 15 & 36,67 \\
\hline Ver & 18 & 3,91 & Normal & 11 & 4,96 & Medir & 15 & 35,83 & Hipertensão & 16 & 3,96 & Pegar & 14 & 37,16 \\
\hline Exercício & 13 & 22,05 & Voltar & 10 & 42,97 & Alguma & 15 & 20,25 & Problema & 14 & 11,99 & Farmácia & 13 & 33,86 \\
\hline Desvantagem & 12 & 17,15 & & & & Sentir & 15 & 15,39 & Normal & 13 & 4,05 & Pagar & 12 & 27,02 \\
\hline Vantagem & 12 & 11,97 & & & & & & & Difícil & 12 & 23,21 & SUS & 11 & 36,17 \\
\hline Realmente & 11 & 8,67 & & & & & & & Pouco & 11 & 10,97 & Dinheiro & 11 & 36,17 \\
\hline Fator & 10 & 22,17 & & & & & & & Ajudar & 10 & 20,71 & Governo & 11 & 31,37 \\
\hline Sua & 10 & 14,21 & & & & & & & Trabalhar & 10 & 10,74 & Complicado & 11 & 23,95 \\
\hline Procurar & 10 & 9,21 & & & & & & & & & & Depender & 10 & 28,06 \\
\hline Cardiologista & 10 & 7,96 & & & & & & & & & & & & \\
\hline
\end{tabular}

Figura 2. Dendograma da Classificação Hierárquica Descendente do corpus "Tratamento".

O primeiro desdobramento se refere a classe 5 chamada de "Aquisição do remédio" foi responsável por $23,72 \%$ dos segmentos de texto. Apareceu predominantemente nas falas de mulheres com maior tempo de diagnóstico. Os principais elementos que se associaram a essa classe foram: remédio, comprar, morrer, conseguir, posto, caro, farmácia, sistema único de saúde (SUS), dinheiro. O conteúdo desta classe trata principalmente sobre o processo e dependência das pessoas em conseguir o remédio e a importância do mesmo. O trecho a seguir ilustra esse contexto: "para a maioria como eu te falei o remedinho é caro, não é barato, e tem muitas pessoas que não tem essas condições para comprar um remédio de cento e poucos reais por mês" (Participante 4, sexo masculino, tempo de diagnóstico 9 anos).

Em seguida surge a classe 1 chamada de "Importância, vantagens e desvantagens" foi responsável por $25,1 \%$ dos segmentos de texto.
Apareceu predominantemente nas falas de homens com pouco tempo de diagnóstico. Os principais elementos que se associaram a essa classe foram: tratamento, seguir, cuidar, nenhum, doença, dificuldade, vantagem, desvantagem, entre outras. O conteúdo desta classe trata principalmente da importância de realizar o tratamento continuamente $\mathrm{e}$ as vantagens e desvantagens associadas a essa rotina. Como pode ser exemplificado:

Então a gente tem que ficar sempre atento é o principal para não ter surpresas, as vantagens são que você consegue ter uma saúde melhor e as desvantagens é que você vai ter que seguir isso para a vida inteira. (Participante 5 , sexo feminino, tempo de diagnóstico 10 anos)

A classe 4 chamada de "Alimentação" foi responsável por $14,23 \%$ dos segmentos de texto. Os principais elementos que se associaram a essa classe foram: sal, remédio, comer, ficar, dei- 
xar, voltar, entre outras. O conteúdo desta classe trata principalmente sobre os cuidados com a alimentação. O trecho a seguir ilustra esse contexto: "dizem que aumenta a pressão, para mim eu levo uma vida normal, como de tudo, lógico sal eu evito, mas é porque eu já fazia a comida não muito salgada desde que eu morava com meu pai" (Participante 17, sexo feminino, tempo de diagnóstico 1 ano).

Na classe 2 chamada de "Sintomas e preocupações", a qual foi responsável por 18,18\% dos segmentos de texto. Apareceu predominantemente nas falas de homens com baixa escolaridade. Os principais elementos que se associaram foram: pressão, tomar, pensar, medicamento, medir, entre outras. O conteúdo desta classe trata dos sintomas e da preocupação relacionada a pressão estar alta, devido a essa preocupação também há muitas ações como medir a pressão a fim de verificá-la, pois os sintomas nem sempre são aparentes. $\mathrm{O}$ trecho a seguir ilustra esse extrato: "um dia você não tem nada e vai medir a pressão ela está lá em cima, no outro dia te dá um sintoma de dor de cabeça e ela não está alta então quando não avisa é pior" (Participante 20, sexo feminino, tempo de diagnóstico 10 anos).

Juntamente com a classe 2, aparece a classe 3 chamada de "Depois da descoberta como ficou a rotina", a qual foi responsável por $18,77 \%$ dos segmentos de texto. Os principais elementos que se associaram a essa classe foram: achar, sempre, dizer, ano, problema, difícil, entre outras. O conteúdo desta classe trata principalmente da rotina modificada pela descoberta da doença, o dia-a-dia do tratamento, suas dificuldades e sentimentos advindos, como exemplificado: "quando eu peguei a receita e o médico falou que eu tinha que tomar esse remédio pelo resto da vida, me assustou, mas hoje em 7 anos tomando o remédio é sem problema nenhum" (Participante 3, sexo masculino, tempo de diagnóstico 6 anos).

Analisando o dendograma do corpus é possível definir que o tratamento é colocado como uma maneira de manter a pressão estabilizada. Os entrevistados comentam sobre as vantagens e desvantagens, os sintomas que a doença apresenta ou não, as preocupações advindas com as consequências de não possuir um controle na pressão, o histórico da descoberta e as mudanças na rotina, incluindo alimentação, exercício físico, a medicação, entre outros. Além disso, há uma culpabilização do governo por não dar a devida atenção à saúde de pessoas que não possuem condições de realizar o tratamento corretamente.

O terceiro corpus a ser analisado pelo software IRAMUTEQ foi sobre as representações sociais tanto do tratamento como da doença, assim uniu-se as duas temáticas em um corpus só. Segundo Camargo e Justo (2013) existem duas maneiras de preparar as linhas de um corpus. A monotemática e a temática, a qual se distingue pela possibilidade da segunda utilizar mais de um tema na mesma análise. A análise de corpus com distintas temáticas nos informa sobre as relações de conteúdo entre os temas, podendo ainda ser usada como uma análise de natureza exploratória (Camargo \& Justo, 2013). No entanto, os autores alertam para a necessidade de se fazer as análises monotemáticas também, pois são elas que aprofundam a compreensão do material estudado. Assim como já realizado anteriormente.

A partir da CHD foram analisados 964 segmentos de texto, retendo $86,61 \%$ do total, gerando 3 classes. O software dividiu o corpus temático em 2 subcorpora, conforme a Figura 3. O primeiro sub-corpus, composto pela classe 3 se refere mais as formas de tratamento, as dificuldades e vantagens de se tratar e relações com a política pública. O segundo sub-corpus formado pela classe 1 e 2 se refere à questões mais práticas, o histórico de como descobriram a doença e o que mudou em relação ao seu tratamento. As classes 1 e 2 se opõem, pois a primeira diz respeito mais à doença, seu histórico e os sentimento que advêm com a descoberta, e a segunda trata-se tanto da doença como do tratamento, relata-se questões práticas da rotina com a doença e quanto do seu consequente tratamento. 


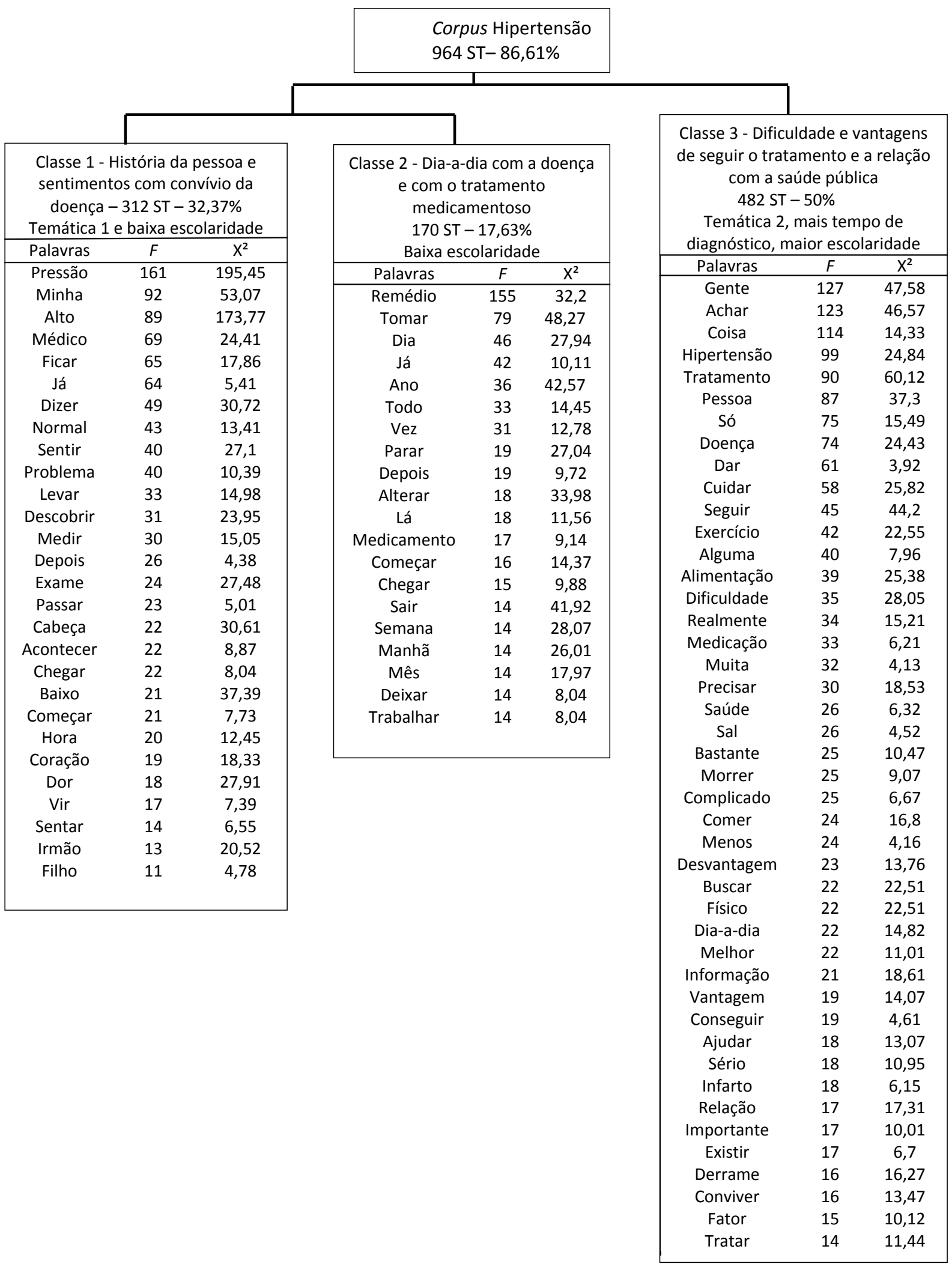

Figura 3. Dendograma da Classificação Hierárquica Descendente da doença e do tratamento.

Assim, primeiramente surge a classe 3 chamada de "Dificuldade e vantagens de seguir o tratamento e a relação com a saúde pública" foi responsável por $50 \%$ dos segmentos de texto.
Apareceu predominantemente nos segmentos de texto relacionados ao tratamento da hipertensão e em pessoas com alta escolaridade e maior tempo de diagnóstico. Os principais elementos que 
se associaram a essa classe foram: hipertensão, tratamento, doença, cuidar, seguir, exercício, alimentação, dificuldade, entre outras. O conteúdo desta classe trata das dificuldades, facilidades, vantagens e desvantagens de seguir o tratamento tanto medicamentoso como não medicamentoso, como a realização de exercícios físicos e uma alimentação saudável. Além disso, houveram falas relacionando as dificuldades encontradas na aquisição do medicamento com a saúde pública. $\mathrm{O}$ trecho a seguir ilustra esse contexto:

. . fazendo uma atividade física e tendo uma boa alimentação eu com certeza não vou morrer disso, a sequencia do tratamento não tem jeito tem que seguir quem não tem condição de fazer essa alimentação, de fazer um esporte e comprar o remédio é complicado. (Participante 3, sexo masculino, tempo de diagnóstico 6 anos)

Em seguida há a classe 1 chamada de "História da pessoa e sentimentos com convívio da doença" foi responsável por $32,37 \%$ dos segmentos de texto. Apareceu predominantemente nos segmentos de texto relacionados à doença da hipertensão e em pessoas com baixa escolaridade. Os principais elementos que se associaram a essa classe foram: pressão, alto, médico, dizer, problema, descobrir, exame, cabeça, dor, entre outras. O conteúdo desta classe trata principalmente de narrativas acerca da descoberta da doença associados com as causas do surgimento, os sintomas sentidos ou não e os sentimentos advindos com a rotina vivida. Conforme o extrato a seguir: "eu fui ficando bem preocupado, então um dia eu fui na empresa medir a pressão e estava bem alta, depois eu procurei um médico e fui me aprofundar mais, lá fiz exames e fui diagnosticado mesmo" (Participante 14, sexo masculino, tempo de diagnóstico 3 anos).

Juntamente com a classe 1 liga-se a ela a classe 2 chamada de "Dia-a-dia com a doença e com o tratamento medicamentoso" foi responsável por $17,63 \%$ dos segmentos de texto. Apareceu predominantemente em pessoas com baixa escolaridade. Os principais elementos que se associaram a essa classe foram: remédio, tomar, dia, ano, vez, todo, vez, parar, entre outras. O conteúdo desta classe trata principalmente da rotina e sua relação com o tratamento medicamentoso. O trecho a seguir ilustra esse contexto: "então a convivência é meio complicada nesse sentido de que todo dia você sabe que você tem que tomar o medicamento tem pessoas que tomam 2 vezes no dia a medicação" (Participante 9, sexo feminino, tempo de diagnóstico 2 anos).

Analisando o dendograma do corpus das duas temáticas percebe-se o entrelaçamento da hipertensão arterial como doença e do tratamento da mesma. As vantagens e desvantagens apresentadas, a relação com a saúde pública, a história de vida e de descoberta da doença e o convívio diário com o tratamento medicamentoso ou não são aspectos presentes.

\section{Discussão}

Ao analisar os segmentos de texto presentes nas falas dos participantes é possível compreender a hipertensão arterial como sinônimo de pressão alta, nas falas dos participantes é recorrente o uso do termo. Assim, a presença freqüente do termo pressão alta encontra correspondência nas produções desenvolvidas nas campanhas de saúde no Brasil, onde o termo é amplamente utilizado (Silva, Bousfield, \& Cardoso, 2013). O principal aspecto observado é a ênfase atribuída ao tratamento medicamentoso. A palavra "remédio" apareceu 155 vezes, possuindo um $c h i^{2}$ de 32,2 no corpus das 2 temáticas juntas. Assim a representação social da hipertensão arterial se associa predominantemente ao controle da doença a partir da medicação. Essa situação encontra concordância em Silva et al. (2013) em que notou-se a centralidade do medicamento como ferramenta para o tratamento e controle da hipertensão.

Além disso, percebe-se uma resistência à utilização de medidas paralelas de controle como a prática regular de atividades físicas e dieta adequada (Costa e Silva \& Moura, 2011). Quando essas práticas são lembradas, há a predominância de conteúdos relacionados ao uso do sal associado à dificuldade em ir a encontros festivos e negar alimentos gordurosos e salgados. Além da relação ao exercício físico percebeu-se um sentimento de culpa por muitos não realiza- 
rem, geralmente pela falta de tempo ou pela não satisfação na realização ou até pela falta de recursos financeiros.

Assim, as mudanças de estilo de vida exigem modificações nos padrões alimentares e práticas sociais já estabelecidas, e isso representa uma mudança na vida diária. Ademais, por se tratar de uma doença crônica, essas mudanças se tornam necessárias para o resto da vida, com isso muitas vezes há a associação de sentimentos negativos frente a doença e o tratamento não medicamentoso torna-se umas das principais dificuldades na efetivação do tratamento (Costa e Silva et al., 2008; Costa e Silva \& Moura, 2011; Figueiredo \& Asakura, 2010; Mantovani et al., 2008; Péres et al., 2003; Silva et al., 2013). As mudanças no contexto socioeconômico, a industrialização, maior acesso a alimentos processados e globalização de hábitos não saudáveis contribuiu para um quadro preocupante sobre as doenças crônicas (Silva et al., 2013).

Tendo em vista a dificuldade nas mudanças de estilo de vida, o tratamento medicamentoso aparece como uma medida "mais fácil", para muitos tomar o "remedinho" todo dia, na mesma hora, se torna um hábito como escovar os dentes, porém há falas que denotam um sentimento negativo ao medicamento, pois possuem a consciência que deverão ingerir a medicação pelo resto da vida.

Em relação à consciência de ser uma doença crônica, e por isso ser para toda a vida, houve divergências nas falas. Alguns participantes possuem falas que denotam sentimentos de tristeza no momento que descobriram que possuíam a doença e que esta seria para toda a vida. Em contrapartida, outros participantes acreditam na possibilidade de um dia pararem de tomar medicação, com esperança de cura. O que corrobora com os dados encontrados na pesquisa de Mantovani et al. (2008), no qual alguns pacientes hipertensos acreditavam na cura da doença a partir de um tratamento bem-sucedido.

Outro aspecto observado é a relação dos sintomas e a aderência ao tratamento. Os participantes se preocupam a partir da presença ou não de seus sintomas, quando possuem alguma dor de cabeça ou dor na nuca, automaticamente pen- sam que a pressão esteja subindo e se preocupam em medir a pressão ou procurar um médico. Há participantes também que não possuem nenhum sintoma, o que ocasiona uma angústia maior ainda pelo fato de nunca saberem se estão ou não com a pressão alta. Dessa forma, a representação social da doença que mais se destaca é a de ser uma doença perigosa e silenciosa, que não se mostra pelos sintomas e pode causar sérias complicações mais tarde como o infarto e o derrame, consequências muito temidas pelos participantes. Assim, as preocupações com os sintomas e com as possíveis consequências tornam-se um recurso para a aderência do tratamento.

Retomando os estudos de Leão e Silva et al. (2013), é possível observar que o desenvolvimento de práticas de controle da hipertensão eram mais frequentes quando havia relato de experiências prévias com algum sintoma ou complicação decorrente da hipertensão arterial. Em contrapartida nas entrevistas realizadas percebeu-se que há uma preocupação tanto em pessoas que possuem sintomas da doença quanto em pessoas que não apresentam sintoma algum. A preocupação torna-se a mesma a partir da consciência de que a pressão pode estar alta sem a presença de nenhum sintoma, aumenta-se assim a angústia de não saber se está alta ou não, aderindo o tratamento de igual forma.

Outro tema discutido pela maioria dos participantes diz respeito aos problemas relacionados ao governo e a saúde pública. Os participantes que possuem uma maior escolaridade acreditam que as pessoas que possuem uma renda baixa tem dificuldades para conseguir a medicação, as consultas e exames necessários. Muitos criticam o governo, o qual em suas opiniões não presta a devida atenção à saúde e oferece os remédios mais baratos e consequentemente mais ineficazes à população. Porém cabe ressaltar que a maioria dos entrevistados possui um nível econômico considerado como "classe média" pelo Instituto Brasileiro de Geografia e Estatística IBGE (www.ibge.gov.br) e dentro dessa realidade frequenta médicos particulares.

Os entrevistados que frequentam os centros de saúde e que possuem renda e escolaridade menores, acreditam que a saúde vem melhoran- 
do. Uma das participantes narrou a sua complicação em um parto há 28 anos, em que nenhum médico em seu pré-natal havia a informado sobre eclampsia e pela falta de informação, quase morreu no parto. Porém a mesma acrescenta que hoje, sempre lhe oferecem as informações necessárias e suas consultas são frequentes. Outra participante comenta que muitas vezes ficou sem o remédio, pois tinha acabado no "postinho", porém agora com o auxílio da Farmácia Popular, nunca mais faltou-lhe o remédio. Com isso, percebe-se uma diferença nas falas entre pessoas com maior e menor escolaridade e renda.

A partir da análise qualitativa dos dados e das classes resultantes da análise dos três corpus, é possível identificar uma sobreposição das representações sociais do tratamento frente a doença. Percebe-se que os conteúdos apresentados nas representações sociais da hipertensão arterial perpassam as questões da rotina, de como foi a descoberta da doença, os sintomas presentes e as preocupações com as consequências da não realização do tratamento. Temas presentes tanto na doença em si, como no tratamento, podendo haver as imbricações entre os temas, afirmando-se que não há ao certo duas representações sociais distintas e sim uma única relacionada ao tratamento abarcando a doença como um todo.

Silva (2014) apresenta que as representações sociais da hipertensão arterial se caracterizam pela forte ligação com o contexto prático que envolve a doença. As modificações no estilo de vida, a incorporação de práticas de controle na rotina do indivíduo se caracteriza na percepção que se dá na doença em si. Para o autor as representações da hipertensão arterial e do tratamento parecem estar intimamente relacionadas, possuindo contexto de sentido bastante semelhantes, centralizados basicamente no contexto prático da doença. Com isso, "parece que ao representar a doença, as pessoas lançam mão também do tratamento para organizar os conhecimentos relacionados ao objeto" (p. 120). A partir da análise dos corpus, comprova-se as interlocuções envolvidas na representação da doença e do tratamento, podendo-se afirmar a existência de uma única representação.

\section{Considerações Finais}

As representações sociais da hipertensão arterial tornam-se questões essenciais para se pensar em novas políticas públicas. As facilidades e dificuldades envolvidas no controle da doença, tanto medicamentosas como não medicamentosas são determinantes para a adesão do tratamento, a qual é permeada pela própria noção que as pessoas possuem de doença.

A partir da pesquisa realizada, conclui-se que a representação social do tratamento da hipertensão arterial é sobreposta à representação da própria doença. As falas sobre a doença adentram temáticas da descoberta, o impacto e as mudanças ocorridas na rotina e a consequente preocupação com as consequências, pensando e agindo para o controle da doença, realizando assim o tratamento. E as representações do tratamento se relacionam com as vantagens, desvantagens, os sentimentos advindos, as relações com o governo, tendo em vista o medo com as consequências da doença. Assim, tratamento e doença se inter-relacionam diretamente, afirmando-se como uma única representação social. Esses indicativos fazem com que ao se pensar em políticas públicas, fatores sociais, psicológicos e ambientais sejam considerados no cuidado de pessoas que vivem com esta condição crônica.

\section{Referências}

Abric, J.-C. (1998). A abordagem estrutural das representações sociais. In A. S. P. Moreira \& D. C. Oliveira (Eds.), Estudos interdisciplinares de representação social (pp. 27-38). Goiânia, GO: AB.

Badziak, R. P. F., \& Moura, V. E. V. (2010). Determinantes sociais da saúde: Um conceito para efetivação do direito à saúde. Revista de Saúde Pública de Santa Catarina, 3(1), 69-79. Recuperado em http://esp.saude.sc.gov.br/sistemas/ revista/index.php/inicio/article/viewFile/51/114

Boell, J. E. W., Meirelles, B. H. S., Silva, D. M. G. V., \& Lessmann, J. C. (2012). Arterial hypertension and diabetes mellitus: Health care in a basic unit. Journal of Nursing, 6(6), 1485-1490. doi:10.5205/01012007. 
Camargo, B. V., \& Justo, A. M. (2013). IRAMUTEQ: Um software gratuito para análise de dados textuais. Temas em Psicologia, 21(2). doi:10.9788/ TP2013.2-16

Chaves, A. M., \& Silva, P. L. (2011). Representações sociais. In L. Camino, A. R. R. Torres, M. E. O Lima, \& M. E. Pereira, Psicologia Social: Temas e teorias (pp. 299-350). Brasília, DF: Technopolitik.

Conselho Nacional de Saúde. (1996). Resolução 196 de 10 de outubro de 1996: Dispõe das diretrizes e normas regulamentadoras de pesquisas envolvendo seres humanos. Brasília, DF: Autor.

Conselho Nacional de Saúde. (2012). Resolução 466 de 12 de dezembro de 2012: Dispõe das diretrizes e normas regulamentadoras de pesquisas envolvendo seres humanos. Brasília, DF: Autor.

Costa e Silva, M. E. D., Barbosa, L. D. da C. S., Oliveira, D. da S., Gouveia, M. T. de O., Nunes, B. M. V. T., \& Alves, E. L. M. (2008). As representações sociais de mulheres portadoras de hipertensão arterial. Revista Brasileira de Enfermagem, 61(4), 500-507. doi:10.1590/S003471672008000400017

Costa e Silva, M. E. D., \& Moura, E. B. M. (2011). Representações sociais de profissionais de saúde sobre hipertensão arterial: Contribuições para a enfermagem. Escola Anna Nery Revista de Enfermagem, 15(1), 75-82. doi:10.1590/S141481452011000100011

D’Ancona, M. A. (1996). Metodologia cuantitativa: estratégias e técnicas de investigación social. Madrid: Síntesis.

Figueiredo, N. N., \& Asakura, L. (2010). Adesão ao tratamento anti-hipertensivo: Dificuldades relatadas por indivíduos hipertensos. Acta Paulista de Enfermagem, 23(6), 782-787. doi:10.1590/ S0103-21002010000600011

Galand, C., \& Salès-Wuillemin, E. (2009). Apports de l'étude des représentations sociales dans le domaine de la santé. Sociétés, 105(3), 35-44.

Ghiglione, R., \& Matalon, B. (1993). O inquérito Teoria e prática. Oeiras, Portugal: Celta.

Jardim, P. C. B. V., Souza, A. L. L., \& Monego, E. T. (1996). Atendimento multiprofissional ao paciente hipertenso. Medicina, 29, 232-238. doi:10.11606/issn.2176-7262.v29i2/3p232-238

Jodelet, D. (2001). As representações sociais. Rio de Janeiro, RJ: Editora da Universidade do Estado do Rio de Janeiro.
Leão e Silva, L. O., Dias, C. A., Rodrigues, S. M., Soares, M. M., Oliveira, M. A., \& Machado, C. J. (2013). Hipertensão Arterial Sistêmica: Representações sociais de idosos sobre a doença e seu tratamento. Cadernos de Saúde Coletiva, 21(2), 121-128. doi:10.1590/S1414$-462 \times 2013000200004$

Mantovani, M. F., Ulbrich, E. M., Pinotti, S., Giacomozzi, L. M., Labronici, L. M., \& Sarquis, L. M. M. (2008). O significado e a representação da doença crônica: Conhecimento do portador de hipertensão arterial acerca de sua enfermidade. Cogitare Enfermagem, 13(3), 336-342. doi:10.5380/ce.v13i3.12964

Ministério da Saúde. (2006). Hipertensão arterial sistêmica. Brasília, DF: Autor.

Ministério da Saúde. (2011). Plano de ações estratégicas para o enfrentamento de doenças crônicas não transmissiveis (DCNT) no Brasil 20112022. Brasília, DF: Autor.

Ministério da Saúde. (2012). Sistema de Gestão Clínica de Hipertensão Arterial e Diabetes Mellitus da Atenção Básica. Brasília, DF: Departamento de Informática do Sistema Único de Saúde. Recuperado em http://hiperdia.datasus.gov.br/

Ministério da Saúde, \& Organização Pan Americana de Saúde. (2004). Avaliação do plano de reorganização da atenção à hipertensão arterial $e$ ao diabetes mellitus no Brasil. Brasília, DF: Ministério da Saúde.

Morin, M. (2004). Parcours de Santé. Paris: Armand Colin.

Morin, M., Apostolidis, T., Dany, L., Preau, M., \& Spire, B. (2007). Diagnósticos, intervenções e avaliações psicossociais no campo sanitário e social. In A. S. P. Moreira \& B. V. Camargo (Eds.), Contribuições para a teoria e o método de estudo das representações Sociais (pp. 253268). João Pessoa, PB: Editora Universitária da Universidade Federal da Paraíba.

Moscovici, S. (1978). Representação social da Psicanálise. Rio de Janeiro, RJ: Zahar.

Moscovici, S. (1982). On social representation. In J. P. Forgas (Ed.), Social Cognition. London: Academic Press.

Moscovici, S. (2010). Representações sociais: Investigações em psicologia Social. Petrópolis, RJ: Vozes.

Oliveira, K. C. S., \& Zanetti, M. L. (2011). Conhecimento e atitude de usuários com diabetes 
mellitus em um Serviço de Atenção Básica à Saúde. Revista da Escola de Enfermagem da USP, 45(4), 862-868. doi:10.1590/S008062342011000400010

Péres, D. S., Magna, J. M., \& Viana, L. A. (2003). Portador de hipertensão arterial: Atitudes, crenças, percepções, pensamentos e práticas. Revista de Saúde Pública, 37(5), 635-642. doi:10.1590/ S0034-89102003000500014

Pérez Riera, A. R. (2000). Hipertensão arterial: Conceitos práticos e terapêutica. São Paulo, SP: Atheneu.

Ratinaud, P., \& Marchand, P. (2012). Application de la méthode ALCESTE à de "gros" corpus et stabilité des "mondes lexicaux" : analyse du "CableGate" avec IraMuTeQ. In Actes des 11eme Journées Internationales d'Analyse statistique des Données Textuelles (pp. 835-844). Liège, France: Journées internationales d'Analyse statistique des Données Textuelles.

Santos, Z. M. S. A., Frota, M. A., \& Cruz, D. M. (2005). Adesão do cliente hipertenso ao tratamento: Análise com abordagem interdisciplinar. Texto \& Contexto Enfermagem, 14(3), 332-340. doi:10.1590/S0104-07072005000300003

Schmidt, M. I., Duncan, B. B., Azevedo e Silva, G., Menezes, A. M., Monteiro, C. A., Barreto, S. M., ...Menezes, P. R. (2011). Chronic non-communicable diseases in Brazil: Burden and current challenges. Lancet, 377(9781), 1949-1961. doi:10.1016/S0140-6736(11)60135-9
Silva, J. P. (2014). Representações sociais da hipertensão arterial sistêmica e relação com práticas de controle da doença (Dissertação de mestrado, Programa de Pós-Graduação em Psicologia, Universidade Federal de Santa Catarina, Florianópolis, SC, Brasil).

Silva, J. P., Bousfield, A. B. S., \& Cardoso, L. H. (2013). A hipertensão arterial na mídia impressa: Análise da revista Veja. Psicologia e Saber Social, 2(2), 191-203. Recuperado em http:// www.e-publicacoes.uerj.br/index.php/psi-sabersocial/article/view/8793/6661

Sociedade Brasileira de Cardiologia, Sociedade Brasileira de Hipertensão, \& Sociedade Brasileira de Nefrologia. (2010). VI Diretrizes Brasileiras de Hipertensão. Arquivos Brasileiros de Cardiologia, 95(1), 1-51. Recuperado em http://publicacoes.cardiol.br/consenso/2010/Diretriz_hipertensao_associados.pdf

Spink, M. J. (1992). Psicologia da saúde: A estruturação de um novo campo do saber. In F. C. B. Campos (Ed.), Psicologia e saúde: Repensando práticas (pp. 11-23). São Paulo, SP: Hucitec. 